\title{
Vibrational resonance in an inhomogeneous medium with periodic dissipation
}

\author{
T. O. Roy-Layinde,,${ }^{1,2}$ J. A. Laoye,${ }^{1}$ O. O. Popoola, ${ }^{2}$ U. E. Vincent $,{ }^{3,4}, *$ and P. V. E. McClintock ${ }^{3}$ \\ ${ }^{1}$ Department of Physics, Olabisi Onabanjo University, Ago-Iwoye, Nigeria \\ ${ }^{2}$ Department of Physics, University of Ibadan, Ibadan, Nigeria \\ ${ }^{3}$ Department of Physics, Lancaster University, Lancaster LA1 4YB, United Kingdom \\ ${ }^{4}$ Department of Physical Sciences, Redeemer's University, Ede, Nigeria
}

(Dated: August 24, 2017)

\begin{abstract}
The role of nonlinear dissipation in vibrational resonance (VR) is investigated in an inhomogeneous system characterized by a symmetric and spatially-periodic potential, and subjected to non-uniform, state-dependent, damping and a bi-harmonic driving force. The contributions of the parameters of the high frequency signal to the system's effective dissipation are examined theoretically in comparison to linearly-damped systems, for which the parameter of interest is the effective stiffness in the equation of slow vibration. We show that the VR effect can be enhanced by varying the nonlinear dissipation parameters; and that it can be induced by a parameter that is shared by the damping inhomogeneity and the system potential. Furthermore, we have apparently identified the origin of the nonlinear-dissipation-enhanced response: we provide evidence of its connection to an Hopf bifurcation, accompanied by monotonic attractor enlargement in the VR regime.
\end{abstract}

PACS numbers: 05.45.-a, 05.90.+m, 05.45.Xt, 02.60.Cb, 05.45.Ac, 05.45.Pq

\section{INTRODUCTION}

Nonlinear systems are ubiquitous in nature. Their nonlinearity may arise in diverse ways, the most important example being, arguably, nonlinear damping/dissipation [1]. This is of particular interest on account of its wide occurrence and applications including, for example, in plasma science $[2,3]$, Josephson junctions [4-6], the Kramers oscillator [7], nanoelectromechanical and nanomechanical systems [8-12], aerospace structures [13], nonlinear suspension and isolation systems [14], the acoustic nonlinearity of an orifice [15], the cochlear amplifier [16], and in the context of quantum information processing with microwave cavity modes [17].

In nonlinear and quasi-linear models, nonlinear dissipation terms have been shown to influence the system dynamics strongly, leading to emergent phenomena. For instance, the stability of the equilibrium points may be altered, giving rise to limit cycles or inducing bifurcation sequences, chaos or other forms of complex dynamics in certain parameter regimes [1]. Mogilevtsev et al. [18] showed that nonlinear dissipation can be designed to combat the effects of an arbitrarily strong linear loss, for both finite-time intervals and stationary states. In the context of so-called absolute negative mobility (ANM), where particles can surprisingly move against a constant bias, nonlinear systems with non-uniform spacedependent damping in a symmetric potential have been investigated in the presence of noise [19], with a periodic force in place of noise [20]; and in a bi-harmonically driven system [21]. Repeated dispersionless (coherent) motion, as well as a ratchet effect, were reported in a medium with a nonlinear friction coefficient $[22,23]$. In

*Corresponding author:u.vincent@lancaster.ac.uk addition, stochastic resonance [24] and the ratchet effect have been described, based on the co-existence of two dynamical states in parameter space [25]. More recent studies revealed that the performance of an underdamped ratchet can be substantially enhanced by an optimal combination of asymmetry of the potential and system inhomogeneity [26]. These results underscore the roles played by noise, bi-harmonic driving and damping inhomogeneity, or by their combination, in enhancing a system's performance and driving it into resonant states [27].

Traditionally, resonance implies a matching of frequencies, e.g. between the natural frequency of an underdamped oscillatory system and a periodic driving force, giving rise to an enhanced response. More recently, however, the term is being used more broadly to describe any case where the amplification or response of a system can be optimised through the adjustment of a parameter that is not necessarily a frequency [27]. In stochastic resonance [28], for example, the parameter in question is the noise intensity and only in special cases [29] is there also any matching of frequencies. In fact, many different kinds of external forces can induce resonances and the latter can manifest in a diversity of different forms, such as chaotic resonance [30, 31], coherence resonance [32, 33], parametric resonance [27], stochastic resonance [28, 34], ghost resonance [35], vibrational resonance [36], autoresonance [27] and anti-resonance [37].

In what follows, we will focus on a form of vibrational resonance (VR). In systems with linear damping, VR has already been widely investigated [38-45] following an early study by Landa and McClintock [36]. The results have shed light on the contributions to the effective potential of the various components of the high frequency signal [27], as well as the roles played by system parameters such as delay and fractional order terms in the induction, enhancement and control of VR [46-48, 4851]. However, relatively little attention has been paid 
to the possible contribution of nonlinear dissipation to the occurrence of VR, as described very recently for a prestressed beam fixed at both ends [52] and for a biharmonically driven plasma [53]. VR is usually discussed in terms of a slowly-driven system's response to variations in the parameters of an imposed fast periodic signal. It has been shown, however, that signal-enhancement or -suppression is also possible through variation of the bifurcation parameters of the model in the presence of a bi-harmonic drive, within appropriate parameter regimes $[53,54]$. This result suggests that internal parameters like frictional inhomogeneity, or nonlinear dissipation, may also play independent or contributory roles in the occurrence of VR.

In this paper, we report the results of an investigation of the role of frictional inhomogeneity in the enhancement, control and induction of vibrational resonance in a driven oscillator with a symmetric potential but nonuniform and space-dependent dissipation. We validate the occurrence of VR both analytically and numerically and provide insights into its origin and into the governing mechanism. We provide clear evidence that VR can be enhanced significantly within appropriate parameter regimes by combining nonlinear dissipation parameters with a bi-harmonic driving force. Furthermore, we show that VR can be induced by a parameter that is shared by the damping inhomogeneity and the system potential. The rest of the paper is organized as follows: the model is introduced and its applications discussed briefly in Sec. II. In Sec. III, an analytic description of the resonance behaviour of the system is presented using the method of separation of motion. Numerical results in Sec. IV are used to verify the theoretical analysis; and conclusions are drawn in Sec. V.

\section{THE MODEL}

The model is a nonlinear dissipative system with a symmetric periodic potential and a periodically-varying friction coefficient, of the generalized dimensionless form

$$
\frac{d^{2} x}{d t^{2}}+\gamma(x) \frac{d x}{d t}+\frac{d V(x)}{d x}=F \cos \omega t+G \cos \Omega t .
$$

The system's periodic potential $V(x)$ is given by

$$
V(x)=-\frac{V_{0}}{k} \cos k x,
$$

while the inhomogeneous damping term $\gamma(x)$ takes the form

$$
\gamma(x)=\gamma_{0}[1-\lambda \sin (k x+\phi)] .
$$

Using Eq. (2) and Eq. (3) in Eq. (1), the system can be written explicitly as

$\ddot{x}+\gamma_{0}[1-\lambda \sin (k x+\phi)] \dot{x}+V_{0} \sin k x=F \cos \omega t+G \cos \Omega t$, where the dots denote differentiation with respect to time $t$. In Eq. (4), $x, \dot{x}$ and $\ddot{x}$ refer to the spatial coordinate of the system at time $t$, the friction term, and the inertial term respectively. The parameter $\gamma(x)$ characterizes the system's dissipation; the damping term is a function of $\gamma_{0}, \lambda$, and $\phi$ which are respectively the amplitude of the damping coefficient, the strength of the system inhomogeneity, and the phase lag between the potential and the damping coefficient which share the same period. $F \cos \omega t$ is a weak periodic driving force of low frequency $\omega$ and amplitude $F$; while $G \cos \Omega t$ is a fast periodic force of high frequency $\Omega(\Omega \gg \omega)$.

The system (4) represents an archetypical model of an inhomogeneously-damped, one-dimensional, single particle evolving within a periodic potential under the influence of a bi-harmonic driving signal - each drive corresponding to a separate, unbiased, external force. The system can equally be adapted to apply to other physical applications such as Josephson junctions, plasmas, phase-locked loops, or pendulums [19-21, 55, 56]. In a Josephson junction set-up, for example, it describes the one-dimensional motion of a charged particle subject to a periodically-varying friction $\gamma(x)$, where $\gamma(x)$ is analogous to the ratio of the conductivities associated with the Cooper-pair and quasiparticle tunneling respectively ([23] and references therein); and $\gamma_{0}$ is a constant damping coefficient such that $\gamma_{0}=v\left(\omega_{j} R C\right)^{-1}[4,5]$ where $\omega_{j}$ is the Josephson frequency and $R$ and $C$ are respectively the resistance and capacitance of the junction. In the special case, $\lambda=0, k=1, V_{0}=1$, the symmetry-breaking requirement met by $\gamma(x)$ - essential for achieving the ratchet effect - is absent, and Eq. (4) then reduces to

$$
\ddot{x}+\gamma_{0} \dot{x}+\sin x=F \cos \omega t+G \cos \Omega t,
$$

which is the well-known linearly-damped pendulum for which VR has already been studied within a range of different parameter regimes [57]. In our analysis of VR in the system (1), we focus on the overdamped regime in which only periodic and quasiperiodic motions are admissible.

\section{THEORETICAL ANALYSIS}

Here, we employ the method of direct separation of the dynamics into fast and slow motions. We thus obtain a set of integro-differential equations, one of which is the equation of slow motion of the system whose response can be modulated by varying the parameters of the highfrequency input drive. The response amplitude, $Q$ given as the ratio of the amplitude $A$ to the frequency $F$ is obtained by solution of the equation for the slow motion. Thus, we consider the solution $x(t)$ of the system (4) as a superposition of only the solutions $\chi(t)$ of slow evolution with frequency $\omega$ and $\psi(t)$ of the fast oscillations with frequency $\Omega$ when $\Omega \gg \omega$, in the form:

$$
x(t)=\chi(t)+\psi(t, \Omega t) .
$$


$\chi(t)$ is periodic with period $T=\frac{2 \pi}{\omega}$ while $\psi$ is periodic in the fast time $\tau=\Omega t$ with period $2 \pi$ and its mean value with respect to fast time $\tau$ is given by

$$
\langle\psi\rangle=\frac{1}{2 \pi} \int_{0}^{2 \pi} \psi d \tau=0 .
$$

The next step is to derive a system of 2-coupled integrodifferential equations for the variables $\chi$ and $\psi$ from the differential equation (4), though the object of main interest is of course the slow component. The first of these equations is obtained by substituting Eq.(6) in Eq.(4) to yield

$$
\begin{aligned}
\ddot{\chi}+\ddot{\psi}+\gamma_{0}[1-\lambda \sin (k \chi+\phi) \cos k \psi-\lambda \cos (k \chi+\phi) \sin k \psi](\dot{\chi}+\dot{\psi})+ & V_{0} \sin k \chi \cos \psi+V_{0} \cos k \chi \sin \psi \\
& =F \cos \omega t+G \cos \Omega t
\end{aligned}
$$

By averaging both sides of Eq. (8) over the period of fast time $\left[0, \frac{2 \pi}{\Omega}\right]$, and noting that $\psi$ is a rapidly-oscillating periodic function of the fast time, so that we can then apply Eq.(7) to write

$$
\begin{aligned}
\ddot{\chi}+\gamma_{0}[1-\lambda \sin (k \chi+\phi)\langle\cos k \psi\rangle-\lambda \cos (k \chi+\phi)\langle\sin k \psi\rangle] \dot{\chi}+ & V_{0} \sin k \chi\langle\cos k \psi\rangle+V_{0} \cos k \chi\langle\sin k \psi\rangle \\
& =F \cos \omega t+\langle G \cos \Omega t\rangle .
\end{aligned}
$$

Simplifying Eq. (9) using the mean values,

$$
\begin{aligned}
\langle G \cos \Omega t\rangle & =0, \\
\langle\sin k \psi\rangle & =\frac{1}{2 \pi} \int_{0}^{2 \pi} \sin k \psi d \tau=0, \\
\langle\cos k \psi\rangle & =\frac{1}{2 \pi} \int_{0}^{2 \pi} \cos k \psi d \tau=J_{0}\left(k \psi_{0}\right),
\end{aligned}
$$

where $\psi_{0}$ is the amplitude of the steady state solution of $\psi$ and $J_{0}\left(\psi_{0}\right)$ is the zeroth-order Bessel function of the first kind, gives

$$
\begin{aligned}
\ddot{\chi}+\gamma_{0}[1 & \left.-\lambda J_{0}\left(k \psi_{0}\right) \sin (k \chi+\phi)\right] \dot{\chi} \\
+ & V_{0} J_{0}\left(k \psi_{0}\right) \sin k \chi=F \cos \omega t .
\end{aligned}
$$

Eq. (11) is the first of the set of coupled equations for the variable $\chi$. The equation for the fast motion $\psi$ is obtained by subtracting Eq. (11) from Eq. (8) and, by using the inertial approximation $\ddot{\psi} \gg \dot{\psi} \gg \psi$, can be approximated to that of a damped and periodically-driven particle whose long-term solution is periodic in fast time $\tau=\Omega t$ by considering that $\psi$ is rapidly oscillating:

$$
\ddot{\psi}+\gamma_{0} \dot{\psi}=G \cos \Omega t
$$

which has steady state solution

$$
\begin{gathered}
\psi=\psi_{0} \cos (\omega t+\theta)=\frac{G}{\Omega \sqrt{\Omega^{2}+\gamma^{2} 0}} \cos (\omega t+\theta) ; \\
\sin \theta=\frac{-\gamma_{0}}{\sqrt{\Omega^{2}+\gamma^{2}}}, \quad \cos \theta=\frac{-\Omega}{\sqrt{\Omega^{2}+\gamma^{2} 0}} .
\end{gathered}
$$

$\psi_{o}$ may be approximated by assuming $\Omega \gg \gamma_{0}$, so that

$$
\psi_{0}=\frac{G}{\Omega^{2}} .
$$

Eq. (11) can now be written as

$$
\begin{gathered}
\ddot{\chi}+\gamma_{0}\left[1-\lambda J_{0}\left(\frac{k G}{\Omega^{2}}\right) \sin (k \chi+\phi)\right] \dot{\chi} \\
+V_{0} J_{0}\left(\frac{k G}{\Omega^{2}}\right) \sin k \chi=F \cos \omega t .
\end{gathered}
$$

Eq. (16) is the analytic expression that we seek for the slow vibration, containing the parameters of the fast signal, and it will be used to compute the theoretical response amplitude $Q$ of the system at the lower frequency $\omega$ by linearising it around the equilibrium points. Eq. (16) can be considered as a forced slow motion of a particle of form

$$
\ddot{\chi}+\gamma_{\mathrm{eff}} \dot{\chi}+\frac{d V_{\mathrm{eff}}}{d \chi}=F \cos \omega t,
$$

where

$$
V_{\mathrm{eff}}=-\frac{V_{0}}{k} J_{0}\left(\frac{k G}{\Omega^{2}}\right) \cos k \chi
$$

and

$$
\gamma_{\mathrm{eff}}=\gamma_{0}\left[1-\lambda J_{0}\left(\frac{k G}{\Omega^{2}}\right) \sin (k \chi+\phi)\right]
$$

are the effective potential and the effective dissipation, respectively. The effective potential, $V_{\text {eff }}(\chi)$ is a function of the parameters $\Omega$ and $G$, and its shape and number of local minima and maxima are determined by the these parameters. Fig. 1(a) plots $V_{\text {eff }}(\chi)$ for $G=0,100,500$ and 750 , with $k=1, \Omega=13$. It is clearly evident that, for all $G$ values, the depth of the well is reduced by increasing the value of $G$ while the shapes remain locally 


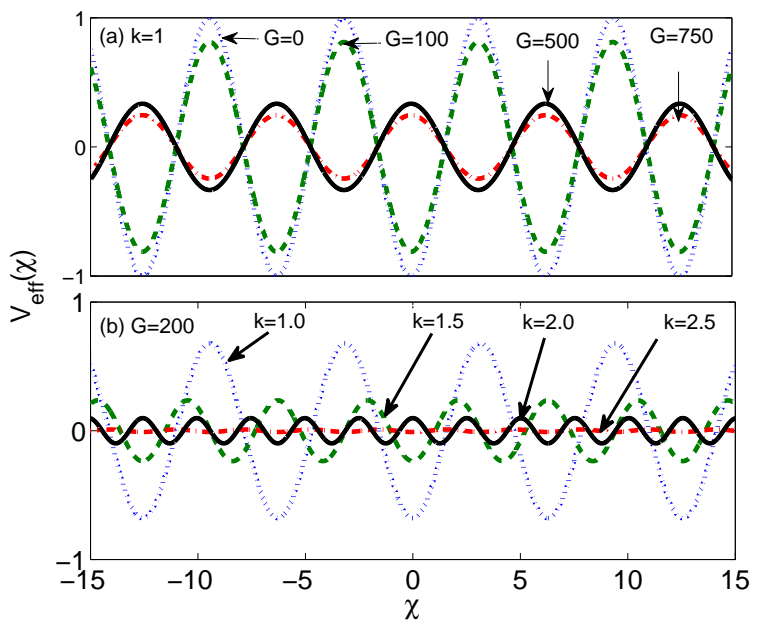

FIG. 1: [Color Online] The effective potential, $V_{\text {eff }}(\chi)$ for: (a) four values of $G$ with $k=1$ and $\Omega=13$; and (b) four values of $k$ with $G=300$ and $\Omega=13$

the same in each case. However, the positions of the maxima for $G=0$ and 100 correspond to those of the minima for $G=500$ and 750 . This remarkable change occurs at $G \simeq 405$. Above and below this value of $G$, the positions of the extrema remain unchanged as $G$ is varied. Fig. 1(b) shows the effective potential $V_{\text {eff }}(\chi)$ for $k=1,1.5,2.0$ and 2.5. Variation in the $k$ value exerts a similar effect on the effective potential to that exerted by $G$ in Fig. 1(a), as would be expected from Eq. (18). It is therefore reasonable to assume that for the system (4), whose vibration can be completely described by Eq. (16), the effect of varying the amplitude $G$ of the high frequency signal can be mimicked by appropriate adjustment of the system parameter $k$. Consequently, any optimisation of signal amplification or suppression achievable through modulation of the parameters of the fast driving force may equally be achieved by adjustment of the system parameter $k$. Note that with $k$ acting like a coefficient for the ratio $\frac{G}{\Omega^{2}}$ in Eq. (18), amplification of the system's response (governed by the ratio of $G$ and $\Omega$ in VR [57]), can effectively be tuned to resonance for fixed parameters of the fast periodic driving signal. This means that the system's response can be controlled effectively by adjustment of its dissipation term to drive it into the resonant state. This fact will be of central importance in what follows.

Next, linearization of the equation of slow oscillation (16) around the equilibrium points $\left(\chi^{*}, \dot{\chi}^{*}\right)$ is carried out using an appropriate approximation to obtain an analytic response amplitude $Q$ to be compared with the $Q$ obtained by computing the full equation of the system (4) numerically in Sec. IV. By re-writing Eq. (17) as a set of coupled first order differential equations without the external driving force in $\left(\chi, \zeta=\frac{d \chi}{d t}\right)$, and then substituting in $\left(\frac{d \chi}{d t}=0, \frac{d \zeta}{d t}=0\right)$ to obtain $\left(0=\chi^{*}, 0=V_{o} J_{o} \sin k \chi^{*}\right)$ where $J_{o}=J_{o}\left(\frac{k G}{\Omega^{2}}\right)$, the equilibrium points around which

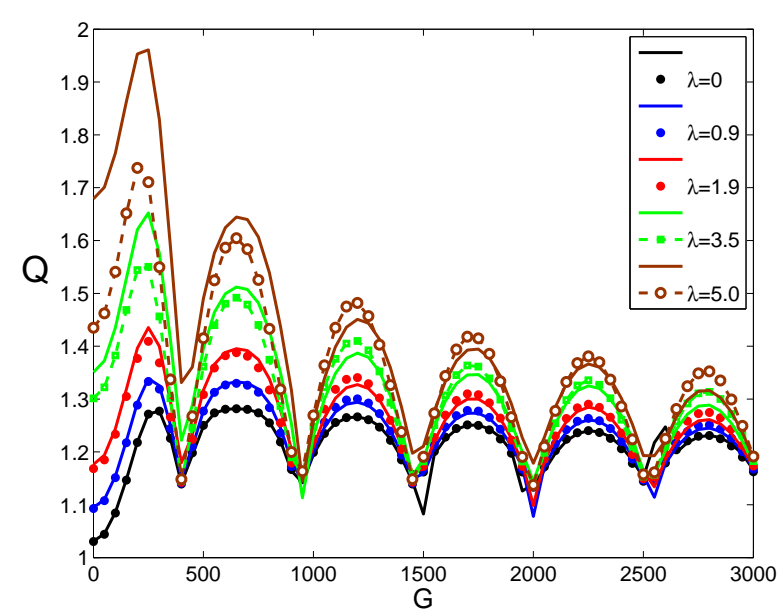

FIG. 2: [Color Online] Dependence of the response amplitude $Q$ on the control parameter $G$ for five values of $\lambda$ (from bottom to top against the ordinate axis, $\lambda=0,0.9,1.9,3.5,5.0$ ). Other parameters are: $\gamma_{0}=1.2, \phi=0.1, k=1.0, F=0.1$, $\omega=0.65$ and $\Omega=13$. Continuous curves represent the numerically-computed $Q$ from Eq. (29) using Eq. (25), while the analytically calculated $Q$ from Eq. (24) are indicated by marker points and(or) broken lines.

slow oscillation occur are $\chi_{\min (\max )}^{*}=2 n \pi$, where $n$ is an integer. The system's oscillation can be described in terms of the deviation of slow motion $\chi$ from the equilibrium points $\chi^{*}$ by using the deviation variable $Y=\chi-\chi^{*}$ in Eq. (16). The equation of motion then becomes

$$
\begin{aligned}
\ddot{Y}+\gamma_{0}[1- & \left.\lambda J_{0} \sin (k Y+\phi) \cos k \chi^{*}\right] \dot{Y} \\
& +V_{0} J_{0} \sin k Y \cos \chi^{*}=F \cos \omega t
\end{aligned}
$$

For $\chi^{*}=\chi_{\min (\max )}, J_{0} \cos k \chi^{*}=\left|J_{0}\right|$ and Eq. (20) can then be written as

$\ddot{Y}+\gamma_{0}\left[1-\lambda\left|J_{0}\right| \sin (k Y+\phi)\right] \dot{Y}+V_{0}\left|J_{0}\right| \sin k Y=F \cos \omega t$.

For $F \ll 1,|Y| \ll 1$ and $\phi \ll 1, \sin (k Y+\phi) \approx(k Y+\phi)$ and $\sin k Y \approx k Y$, so that Eq. (21) becomes

$$
\ddot{Y}+\gamma_{0}\left[1-\lambda\left|J_{0}\right|(k Y+\phi)\right] \dot{Y}+V_{0}\left|J_{0}\right| k Y=F \cos \omega t .
$$

Clearly, the damping term in system (22) consists of both linear $\gamma_{0}\left[1-\lambda \phi\left|J_{0}\right|\right] Y$ and nonlinear $\left[\gamma_{0} \lambda k\left|J_{0}\right|\right] Y \dot{Y}$ contributions. By neglecting the nonlinear damping term, an approximate damped and periodically forced linear equation of the form

$$
\ddot{Y}+\gamma_{L} \dot{Y}+\omega_{r}^{2} Y=F \cos \omega t
$$

is obtained; where the linear damping coefficient is $\gamma_{L}=$ $\gamma_{0}\left(1-\lambda \phi\left|J_{0}\right|\right)$, the resonant frequency is $\omega_{r}=\sqrt{V_{0}\left|J_{0}\right| k}$ and its steady state solution $Y(t)$ which describes the ultimate behaviour of the system in the long-time limit $t \rightarrow \infty$ is $Y(t)=A_{L} \cos (\omega t+\Phi) ; A_{L}=\frac{F}{\sqrt{S}}, S=W^{2}+$ $\gamma_{L}^{2} \omega^{2}$ and $W=\omega_{r}^{2}-\omega^{2}$. The response amplitude is given 


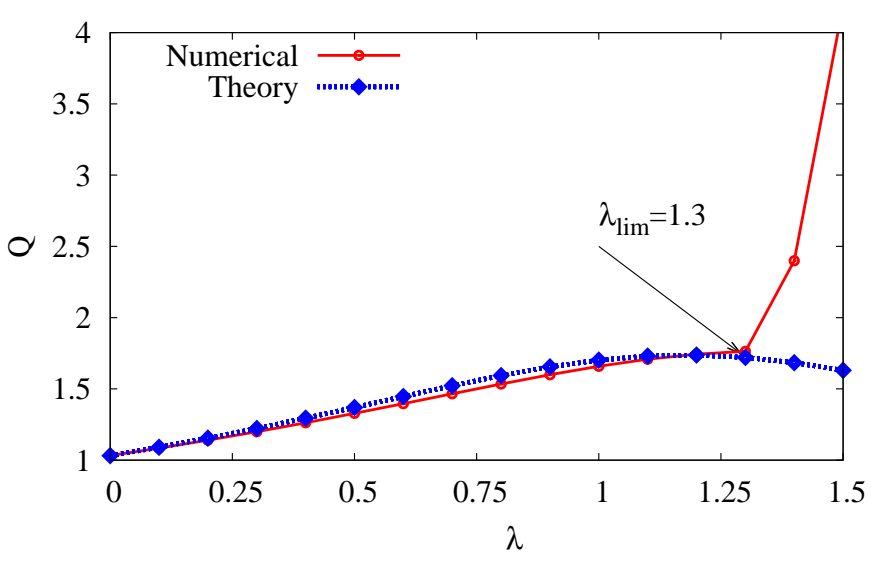

FIG. 3: [Color Online] Comparison between theory and numerics for the dependence of the response amplitude $Q$ on the coefficient of nonlinear dissipation $\lambda$ for $G=15$. Other parameters are: $\gamma_{0}=1.2, \phi=0.85, \omega=0.65$ and $\Omega=13$. The continuous curve (red) represents the numerically-computed $Q$ from Eq. (29) using Eq. (25), while the theoretically calculated $Q$ from Eq. (24) is indicated by the broken line with marker points in blue.

by the factor $Q$ which is defined as the ratio between the output and the forcing signals:

$$
Q=\frac{A_{L}}{F}=\frac{1}{\sqrt{W^{2}+\gamma_{L}^{2} \omega^{2}}} .
$$

In Eq. (24), $Q$ is maximum when $S$ is minimum, i.e. at resonance $W=0$ or $\omega_{r}=\omega$. Note that the damping term $\gamma_{L}$ is a function of the fast motion parameters $G$ and $\Omega$ and other parameters $(k, \lambda, \phi)$ of the damping inhomogeneity. The implication is that in the system (23), considerable variation in the system's response can indeed be achieved by varying certain parameters of the damping term. From the theoretical response amplitude, we deduce that the value of $\lambda$ corresponding to $Q_{\max }$ is given as $\lambda_{\max }=\frac{1}{\phi\left|J_{0}\right|}$. Thus, for $\lambda>\lambda_{\max }$, the system's response, $Q$ diminishes.

\section{NUMERICAL RESULTS}

To compute the system's response to the biharmonic forcing, a convenient approach is to express Eq. (4) as a system of two first-order autonomous ordinary differential equations (ODEs):

$$
\begin{aligned}
\frac{d x}{d t}= & y, \\
\frac{d y}{d t}= & -\gamma_{0}[1-\lambda \sin (k x+\phi)] \dot{x}-V_{0} \sin k x \\
& +F \cos \omega t+G \cos \Omega t .
\end{aligned}
$$

Numerical integration of Eq. (25) is performed in the overdamped regime using a fourth-order Runge-Kutta scheme with step size $\Delta t=0.01$ over a simulation time interval $T_{s}=n T$ with $T=\frac{2 \pi}{\omega}$ being the period of the oscillation where $\omega$ is the low frequency input signal and $n(=1,2,3 \ldots)$ is the number of complete oscillations. We used zero initial conditions, a relaxation time of $100 T$ and fixed the values of the potential amplitude and drive parameters at $V_{0}=1, F=0.1, \omega=0.65$ and $\Omega=13$. These choices ensure that the system is in the overdamped regime in which only periodic or quasiperiodic motion is admissible. The other parameters, $\gamma, \lambda$, $\phi$ are chosen within a regime so as to optimize the emergence of VR for $n=200$.

\section{A. Occurrence of VR}

Our main objective here is to solve for the response amplitude at frequency $\omega$, because it provides an idea of how the low frequency signal is amplified by the high frequency signal, thereby characterizing VR. The response is computed from the amplitudes $B_{s}$ and $B_{c}$ of the Fourier spectrum of the output signal, where $B_{s}$ and $B_{c}$ are

$$
\begin{aligned}
& B_{s}=\frac{2}{n T} \int_{0}^{n T} x(t) \sin \omega t d t \\
& B_{c}=\frac{2}{n T} \int_{0}^{n T} x(t) \cos \omega t d t .
\end{aligned}
$$

Conventionally, the amplitude is given by,

$$
A=\sqrt{B_{s}^{2}+B_{c}^{2}} .
$$

and the phase shift as,

$$
\Phi=\tan ^{-1}\left(\frac{Q_{s}}{Q_{c}}\right) .
$$

The response amplitude is thus given as

$$
Q=\frac{A}{F}=\frac{\sqrt{B_{s}^{2}+B_{c}^{2}}}{F} .
$$

We begin by examining the dependence of frictional inhomogeneity on the occurrence of VR. Shown in Fig. 2 is the response amplitude $Q$ as a function of the amplitude of the high frequency signal, for five values of the dissipation parameter $\lambda$. The values of $Q$ calculated analytically from Eq. (24) (marker points and dashed lines) are compared with numerical values (continuous curves) computed from Eq. (29) for $\lambda=0, \lambda=0.9, \lambda=1.9$, $\lambda=3.5$ and $\lambda=5.0$. Note that the case for $\lambda=0$, reproduced here for comparison and consistency was reported earlier by Rajasekar et al. [57]. Whereas at lower values of $\lambda(0,0.9,1.9)$, the agreement is excellent; as $\lambda$ becomes large (e.g., $\lambda=3.5$ and 5.0, as shown) the theoretical and numerical results no longer agree so well, although the essential features of the curves, including the shape and trend remain in good agreement. In order to 


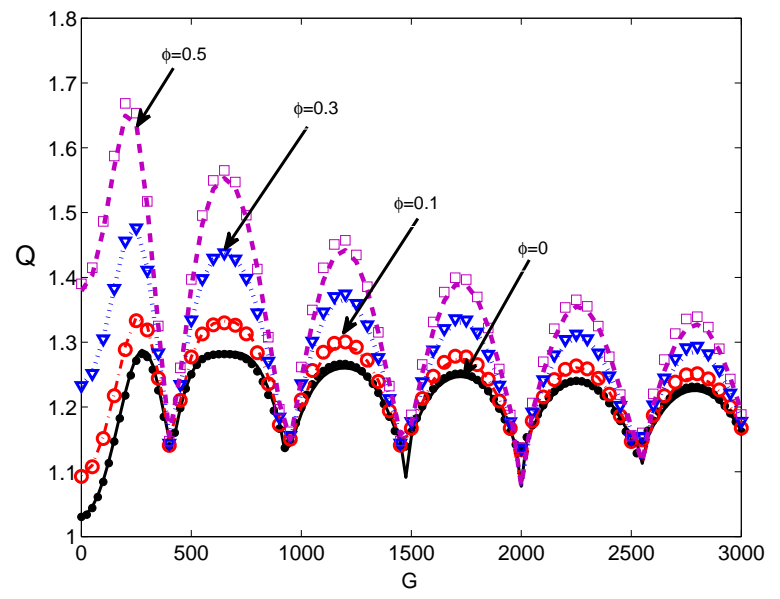

FIG. 4: [Color Online] Dependence of the response amplitude $Q$ on the control parameter $G$ for four values of phase shift: from bottom to top $\phi=0,0.1,0.3$ and 0.5. Other parameters are: $\gamma_{0}=1.2, \lambda=0.9, k=1.0, F=0.1, \omega=0.65$ and $\Omega=13$. The continuous and dashed curves represent the numerically-computed $Q$ from Eq. (29) using Eq. (25), while the corresponding analytically calculated $Q$ from Eq. (24) are indicated by marker points (solid shapes) of the same colour.

ascertain the limit of validity of the theoretical approach, we plot in Fig. 3 the numerical and theoretical response curves, $Q$ both as functions of the nonlinear dissipation coefficient, $\lambda$, where $\lambda_{\text {lim }}$ denotes the theoretical limit of validity. For $\lambda<\lambda_{\text {lim }}=1.3$, the curves are in excellent agreement, whereas for $\lambda>\lambda_{\text {lim }}=1.3$, the deviation of the numerical $Q$ from the theoretical $Q$ grows. This discrepancy may be attributed to the approximation of the damping term in Eq. (22) where we had dropped the nonlinear component of the damping, $\left[\gamma_{0} \lambda k\left|J_{0}\right|\right] Y \dot{Y}$ to obtain the linear equation given by Eq. (23). Intuitively, the low dissipation regime of $\lambda$ for which agreement exist corresponds to the experimentally significant regime where better device response could be obtained at high frequency signals [58]. In general, low dissipation is desirable, since it allow for more device efficiency and sensitivity, as well as less susceptibility to mechanical noise and wear [59]. A remarkable feature is the occurrence of multi-resonance for varying $G$ in the presence of the slow signal even when the system is linearly damped [57], that is, for $\lambda=0$ as shown Fig. 2. However, the increase in the strength of the nonlinear dissipation coefficient $\lambda$ produces a monotonic enhancement in the system's response at each resonance point. Here, resonances occur for small values of $Q_{\max }$ for $\omega=0.65, \Omega=13, F=0.9$, $k=1$ and $\phi=0.1$. Moreover, the trend of $Q$ curves for the nonlinearly damped system $(\lambda>0)$ compared with the linearly damped $\operatorname{system}(\lambda=0)$ shows that as the strength of nonlinearity is increased, the differences between maxima of the response amplitude is magnified and the effect becomes more evident at lower values of G.
Further evidence for the possibility of enhancing $Q_{\max }$ by variation of a dissipation parameter is presented in Fig. 4, for the response amplitude(s) $Q$ as a function of the amplitude of the high frequency signal for four values of phase shift $\phi$, between the nonlinear dissipation and the periodic potential $(\phi=0, \phi=0.1, \phi=0.3$ and $\phi=0.5)$. The numerically-computed $Q$ from Eq. (29) using Eq. (25) (continuous and broken curves) and their corresponding analytically-calculated $Q$ from Eq. (24) (solid shapes) are in agreement as clearly depicted by the close proximities of the location of the point markers (analytic values) to their corresponding (of the same colour) continuous lines. Monotonic enhancement in the system's response to modulation of the amplitude of the fast drive at resonance, similar to the effects produced by variation of $\lambda$ is observed within a good choice of values of the driving signal parameters $\omega, \Omega$ and $F$. The value of $Q_{\max }$ increases with increasing $\phi$, with the effect being more pronounced at low $G$. Since the damping is nonlinear at $\phi=0$, the response curve at $\phi=0$ bears close resemblance to the behavior of $Q$ at very weak values of the parameter $\lambda$ shown in Fig. 2. For $\phi>0$ presented in Fig. 4, increasing the phase difference $\phi$ produces a significant monotonic increase in $Q_{\max }$ - the effect being more pronounced at lower values of the amplitude $G$ of fast drive. Though multi-resonance is produced by varying $G$ in the presence of the slow signal, the system's response at resonance can be enhanced or suppressed by adjustment of the phase $\phi$. This shows that the dissipation parameters can indeed be used to control the system's response at resonance: the dissipation parameters $\lambda$ and $\phi$ are evidently able to control the resonance, as shown in Figs. 2 and 4.

Considering that the system's potential and dissipation are both periodic in $k$, it implies that resonance can be induced by adjustment of the parameter $k$. The roles played by the ratio of the parameters of the fast signal in the occurrence of VR [57] can be performed/enhanced by $k$ in a multiplicative fashion based on the ratio $k \frac{G}{\Omega}$ in Eq. (16), even when the parameters of the fast signal are fixed. Finally, for fixed values of $G$ and $\omega$, and with variation of $k$, the system can be driven into resonance as shown in Fig. 5 for three values of $G$, namely, $G=$ $250, G=350$ and $G=650$. We have plotted in Fig. 5 the analytic response (filled circles) as obtained from Eq. (24) superimposed on the numerical response (Eq. (29)) obtained from Eq. (25) (curves) to demonstrate the good agreement. In addition, multiple resonance peaks occur at larger values of $G$ as observed in Fig. 5 .

Exploring the VR landscape in parameter space gives a convenient view of the range of system parameters for which one can achieve a strong system response, which is significant for experimental applications. Fig. 6, shows the response $Q$ as a function of both the dissipation parameter $\lambda$ and the amplitude $G$ of the fast signal in the parameter range: $(\lambda, G) \in((0.0,3.0),(0.0,500))$, with $\phi=0.85$, and other parameters fixed as before. Fig. 6 shows in red and green the regimes of strong resonance 


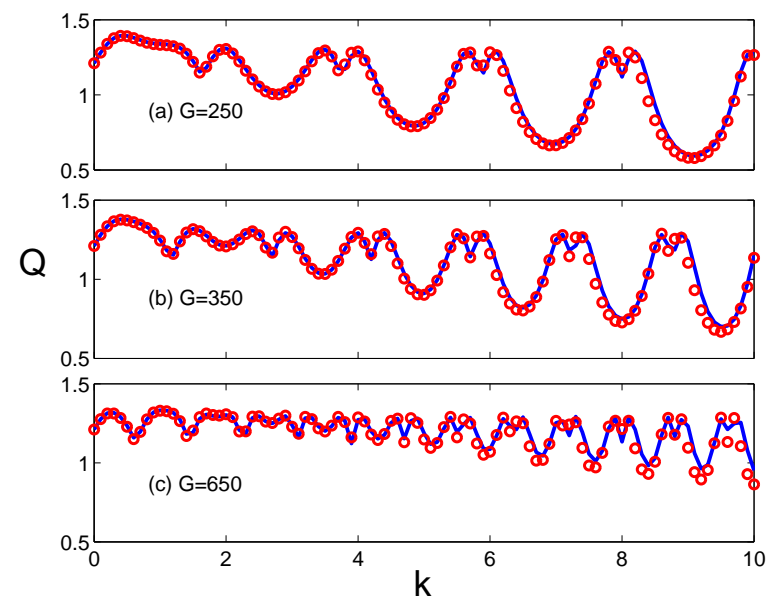

FIG. 5: [Color Online] Dependence of the response amplitude $Q$ on the control parameter $k$ for three amplitudes of fast forcing, $G=250, G=350$ and $G=650$. The other parameters are $\gamma_{0}=1.2, \lambda=0.9, \phi=0.1, F=0.1, \omega=0.65$ and $\Omega=13$. The continuous curves represent the numerically computed $Q$ from Eq. (4) while the corresponding analytically calculated $Q$ from Eq. (24) is shown as open circle (marker) points.

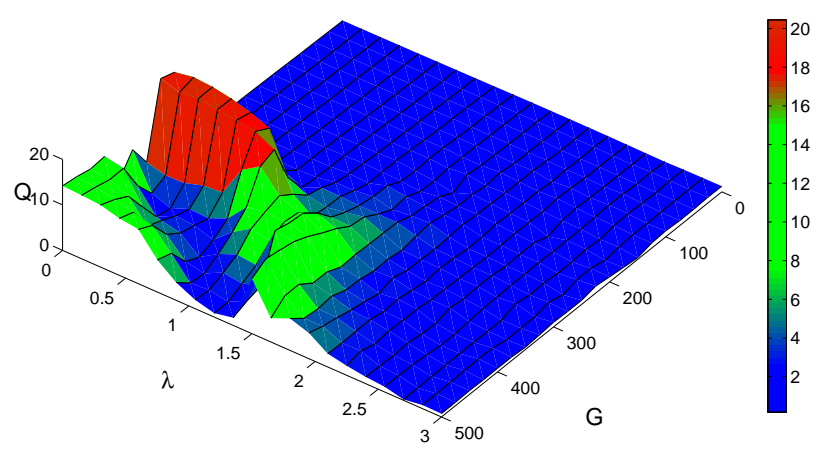

FIG. 6: [Color Online] 3-dimensional plot showing the dependence of the response amplitude $Q$ on the fast signal amplitude $G$ and the dissipation parameter $\lambda$ for a phase shift of $\phi=0.85$. Notice that small values of $G$ also produce some low peaks in the regime marked in deep blue; while the largest peaks occur at higher $G$ and lower $\lambda$.

that can be obtained with weak dissipation (typically, $\lambda<2.0$ ) and high amplitudes of the fast signal (typically, $G>200)$. Elsewhere, however, weak resonances peaks can also appear and are indicated in blue.

\section{B. Origin and mechanism of VR}

We can now seek to understand the origin and mechanism of VR by exploring the underlying dynamics and, in particular, the bifurcation structure and attractors in Poincaré section. Proceeding as before, we calculated the time-asymptotic motion of the system (25), visualizing the trajectories as a series of points in $(x, v)$ phasespace, where $v=\dot{x}$; and recording only one point per period of the external drive, i.e. a point is plotted when $\omega t=\delta+n 2 \pi$ where $\delta$ is the Poincaré phase. Using a parameter of the nonlinear dissipation, in this case $\lambda$, as the bifurcation parameter we first examine the bifurcation structure. It is well known that in nonlinear systems, resonance curves are closely linked to the underlying bifurcation structure $[27,53,60,61]$. In particular, Kozłowski et al. [61] and Roy-Layinde et al. [53] have shown that symmetry-breaking $(s b)$ bifurcations occur between resonances. Here, we report a new dynamical transition mechanism associated with resonance. Fig. 7 shows the forward bifurcation diagram (dotted red) obtained by increasing the value of $\lambda$ from 0 to 4 , the corresponding numerically computed response curve $Q$ (dashed blue line), and the maximal Lyapunov exponent $\lambda_{\max }$ (green line) for $G=15$. There is a clear and striking correlation between the $Q$ plot and $\lambda_{\max }$ at $\lambda_{\mathrm{thr}} \approx 1.30$ just before the Hopf bifurcation takes place for increasing $\lambda$. Preceding the Hopf bifurcation transition point, $H$ (i.e. $\lambda \approx 1.66$ ), the system appears to be in a quiescent state wherein the strength of periodic nonlinear dissipation is incapable of exciting it into resonance. Within this regime, $\lambda_{\max }$ changes values from $\lambda_{\max }<0$ (periodicity) to $\lambda_{\max } \approx 0$ (quasiperiodicity), at $\lambda_{\mathrm{thr}} \approx 1.30$ - a threshold dissipation above which the system is excited into resonance, thereby signaling the onset of VR as shown, with the first resonant peak appearing at $\lambda \approx 1.85$ and in the neighbourhood of the Hopf bifurcation; and thereafter, several resonance peaks emerges. Evidently, for $\lambda>\lambda_{t h r} \approx 1.30$, $Q$ first increases exponentially, and then experiences a sudden jump at the Hopf bifurcation point, $H$. Thus, the jump in $Q$ signalling VR clearly originates from the Hopf bifurcation, thereby strongly suggesting that VR is linked to the bifurcation of the attractors.

To complete the picture, Fig. 8 illustrates the dynamical mechanism accompanying the bifurcation leading to VR, which shows the dynamics emerging from a periodic orbit for $\lambda=0$ to enlarged quasiperiodic orbits as $\lambda$ increases. We have plotted the orbits for five different values of $\lambda$ to illustrate that further increase in $\lambda$ leads to a monotonic increase in the attractor size for other values of $\lambda>0$. It is precisely the growth in attractor size of the attractor with $\lambda$ that is responsible for the occurrence of VR, as characterized by the monotonic enhancement of peaks seen in Fig. 2 for increasing values of $\lambda$.

\section{CONCLUDING REMARKS}

We have investigated the role of nonlinear dissipation in relation to $\mathrm{VR}$ in an inhomogeneous system characterized by a symmetric, spatial, periodic potential and subjected to non-uniform state-dependent damping and 


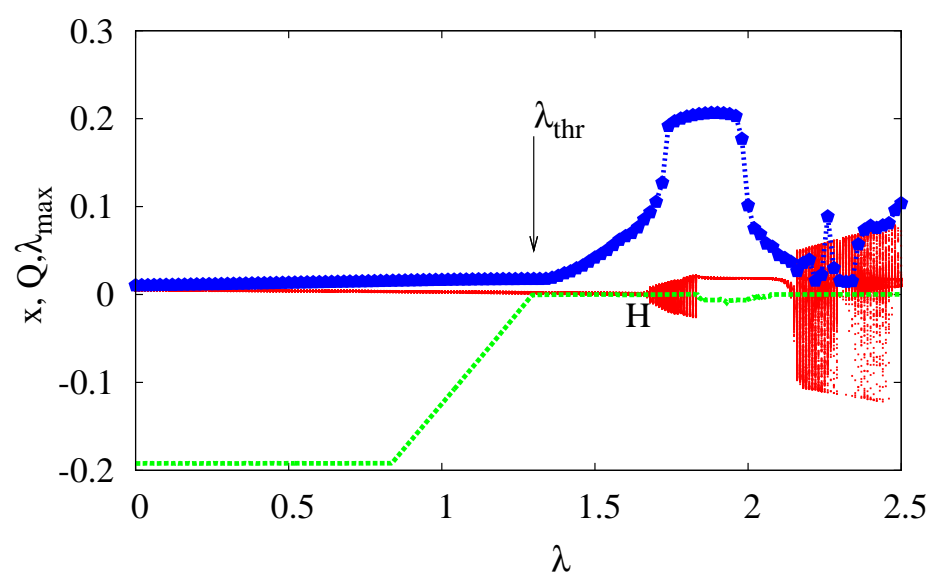

FIG. 7: [Color Online] The bifurcation diagram (dotted red) of the displacement, $x$, the corresponding response curve $Q$ (blue) and maximal Lyapunov exponent, $\lambda_{\max }$, (green) for $G=15$. The other parameters are fixed as follows: $\gamma_{0}=1.2$, $k=1.0, \phi=0.85, V_{0}=1.0, F=0.1, \omega=0.65$, and $\Omega=13$.

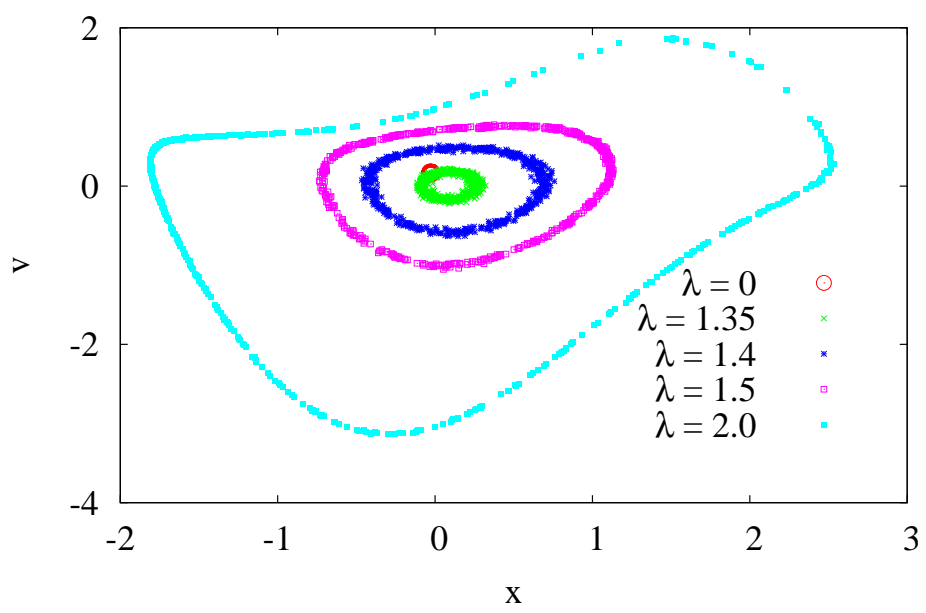

FIG. 8: [Color Online] Transition from periodicity to quasiperiodicity accompanying the onset of VR with variation of the dissipation parameter $\lambda$ : periodic orbit for $\lambda=0$ (red), monotonic quasiperiodic attractor enlargement for $\lambda=1.35$ (green), 1.4(blue), 1.5(magneta), and 2.0 (cyan). The other parameters are fixed at: $G=15, \gamma_{0}=1.2, k=1.0$, $\phi=0.85, V_{0}=1.0, F=0.1, \omega=0.65$, and $\Omega=13$.

a bi-harmonic signal. The contributions from both the parameters of the frictional inhomogeneity and from the fast signal in the resonant state were first identified based on the computed effective potential and the effective dissipation terms, from which we deduced that these could be employed to induce and control resonances in a similar manner to the roles played by delay terms reported earlier $[46,62]$. In contrast to the case of linearly damped systems, where the effective stiffness is the parameter of interest, we examined theoretically and confirmed numerically the contributions of the parameters of the high frequency signal to the system's effective dissipation. We found that, within an appropriate parameter regime, VR can be significantly enhanced by varying the nonlinear dissipation parameters; and that it can be induced by a parameter that is commonly shared by the damping inhomogeneity and the system potential. Scanning the system in the $(\lambda, G)$ two-parameter plane, we found that the regions of optimal resonance corresponds to low values of dissipation $(\lambda<2.0)$ and high values of the fast signal amplitude $(G>200)$; while elsewhere weak resonances occur. The underlying dynamics revealed that the origin of the nonlinear-dissipation-enhanced resonance could be linked to monotonic attractor enlargement arising from a Hopf bifurcation taking place near the onset of VR.

We remark that certain features of vibrational resonance have potential industrial applications including for output filtering, control and enhancement, signal detection, extraction or separation, noise reduction, or highlighting specific elements of a signal. More specifically, advanced technological applications exhibit better performance and efficiency when ratchet-like devices such as sensors, nonlinear mixers, filters, atomic scale imaging and amplifiers are operated in resonant modes - possibilities that can be fully explored in the near future. In controlling the motion of tiny particles in nanoscience or micro or macroscale oscillators [27], one could explore the optimal choice of dissipation parameters and of the fast input signals functioning as controllers, in order to dictate the direction of particle transport and maximise the flux. Finally, we comment that our model could immediately be realised experimentally in an electronic circuit including a Josephson junction with its external drives modeled as input voltage/current sources.

\section{Acknowledgments}

UEV is supported by the Royal Society of London, through their Newton International Fellowship Alumni scheme. The authors are indeed grateful to the reviewers for their constructive comments. The research was supported by the Engineering and Physical Sciences Research Council UK (grant No EP/M015831/1). No new data were created during this study.
[1] S. Elliott, M. Ghandchi Tehrani, and R. S. Langley, Phil. Trans. R. Soc. A 373, 20140402 (2015).

[2] H. G. Enjieu Kadji, B. R. Nana Nbendjo, J. B. Chabi Orou, and P. K. Talla, Phys. Plasmas 15, 032308 (2008).
[3] M. Siewe Siewe, F. Moukam Kakmeni, C. Tchawoua, and P. Woafo, Report 39090566, International Atomic Energy Agency (IAEA), Abdus Salam International Centre for Theoretical Physics, Trieste (Italy) (2007). 
[4] P. Silvestrini, J. Appl. Phys. 68, 663 (1990).

[5] P. Silvestrini, Phys. Rev. B 46, 5470 (1992).

[6] M. Siewe Siewe, H. Cao, and M. Sanjuan, Chaos, Solitons and Fractals 39, 10921099 (2009).

[7] V. V. Semenov, A. B. Neiman, T. E. Vadivasova, and V. S. Anishchenko, Phys. Rev. E 93, 052210 (2016).

[8] A. Eichler, J. Moser, J. Chaste, M. Zdrojek, I. WilsonRae, and A. Bachtold, Nat. Nanotech. 6, 339 (2011).

[9] M. Imboden, O. Williams, and P. Mohanty, Appl. Phys. Lett. 102, 103502 (2013).

[10] M. Imboden, O. A. Williams, and P. Mohanty, Nano Lett. 13, 4014 (2013).

[11] M. Imboden and P. Mohanty, Phys. Rep. 534, 89 (2014).

[12] J. Atalaya, T. W. Kenny, M. L. Roukes, and M. I. Dykman, Phys. Rev. B 94, 195440 (2016).

[13] J. R. Wright and J. E. Cooper, Introduction to Aircraft Aeroelasticity and Loads (John Wiley \& Sons, New York, 2008).

[14] C. Surace, K. Worden, and G. R. Tomlinson, Proc. Inst. Mech. Eng., Part D: J. Automobile Eng. 206, 3 (1992).

[15] U. Ingard and H. Ising, J. Acoust. Soc. Am. 42, 60017 (1967).

[16] E. De Boer, Mechanics of the Cochlea: Modelling Efforts (Springer,New York, NY, 1996).

[17] A. Roy, Z. Leghtas, A. D. Stone, M. Devoret, and M. Mirrahimi, Phys. Rev. A 91, 013810 (2015).

[18] D. Mogilevtsev, A. Mikhalychev, V. S. Shchesnovich, and N. Korolkova, Phys. Rev. A 87, 063847 (2013).

[19] S. Saikia and M. C. Mahato, Physica A 389, 4052 (2010).

[20] C. Mulhern, Phys. Rev. E 88, 022906 (2013).

[21] L. Du and D. Mei, Phys. Rev. E 85, 011148 (2012).

[22] S. Saikia and M. C. Mahato, Phys. Rev. E 80, 062102 (2009).

[23] S. Saikia and M. C. Mahato, J. Phys.: Cond. Matt. 21, 175409 (2009).

[24] S. Saikia, A. M. Jayannavar, and M. C. Mahato, Phys. Rev. E 83, 061121 (2011).

[25] D. Kharkongor, W. L. Reenbohn, and M. C. Mahato, Phys. Rev. E 94, 022148 (2016).

[26] S. Saikia, Physica A 468, 219 (2017).

[27] S. Rajasekar and M. A. F. Sanjuan, Nonlinear Resonances, Springer Series in Synergetics (Springer, Switzerland, 2016).

[28] M. I. Dykman, D. G. Luchinsky, R. Mannella, P. V. E. McClintock, N. D. Stein, and N. G. Stocks, Nuovo Cimento D 17, 661 (1995).

[29] N. G. Stocks, N. D. Stein, S. M. Soskin, and P. V. E. McClintock, J. Phys. A: Math. Gen. 25, L1119 (1992).

[30] L. Y. Chew, C. Ting, and C. H. Lai, Phys. Rev. E 72, 036222 (2005).

[31] S. Nobukawa, H. Nishimura, and T. Yamanishi, Scientific Reports p. 1331 (2017)

[32] A. S. Pikovsky and J. Kurths, Phys. Rev. Lett. 78, 775 (1997).

[33] O. V. Ushakov, H. J. Wünsche, F. Henneberger, I. A. Khovanov, L. Schimansky-Geier, and M. A. Zaks, Phys. Rev. Lett. 95, 123903 (2005).

[34] J. Casado-Pascual, J. Gómez-Ordónez, and M. Morillo, Chaos 15, 26115 (2005).

[35] S. Rajamani, S. Rajasekar, and M. A. F. Sanjun, Comm. Nonlin. Sc. Num. Sim. 19, 4003 (2014).

[36] P. S. Landa and P. V. E. McClintock, J. Phys. A: Math. Gen. 33, L433 (2000).

[37] R. Jothimurugan, K. Thamilmaran, S. Rajasekar, and
M. A. F. Sanjuán, Nonlin. Dyn. 83, 1803 (2016).

[38] M. Gitterman, J. Phys. A: Math. Gen. 34, L355 (2001).

[39] I. Blekhman and P. Landa, Intern. J. Non-Lin. Mech. 39, 421 (2004).

[40] S. Jeyakumari, V. Chinnathambi, S. Rajasekar, and M. A. F. Sanjuan, Chaos 19, 043128 (2009).

[41] Y. Qin, J. Wang, C. Men, B. Deng, and X. Wei, Chaos 21, 023133 (2011).

[42] S. Rajasekar, J. Used, A. Wagemakers, and M. Sanjuan, Commun. Nonlin. Sci. Num. Sim. 17, 3435 (2012).

[43] V. N. Chizhevsky, Phys. Rev. E 89, 062914 (2014).

[44] K. Abirami, S. Rajasekar, and M. Sanjuan, Commun. Nonlin. Sci. Numer. Sim. 47, 370 (2017).

[45] S. Guruparan, V. Ravichandran, V. Chinnathambi, and S. Rajasekar, Ukrainian J. Phys. 62, 51 (2017).

[46] J. H. Yang and X. B. Liu, Chaos 20, 033124 (2010).

[47] J. H. Yang and X. B. Liu, J. Phys. A: Math. Theor. 43, 122001 (2010).

[48] C. Jeevarathinam, S. Rajasekar, and M. A. F. Sanjuan, Phys. Rev. E 83, 066205 (2011).

[49] A. Daza, A. Wagemakers, S. Rajasekar, and M. Sanjuan, Commun. Nonlin. Sci. Num. Sim. 18, 411 (2013).

[50] J. Yang and H. Zhu, Chaos 22, 013112 (2012).

[51] T. L. M. D. Mbong, M. S. Siewe, and C. Tchawoua, Mech. Res. Commun. 78, Part A, 13 (2016).

[52] T. L. M. D. Mbong, M. S. Siewe, and C. Tchawoua, Commun. Nonlin. Sci. Numer. Sim. 22, 228 (2015).

[53] T. O. Roy-Layinde, J. A. Laoye, O. O. Popoola, and U. E. Vincent, Chaos 26, 093117 (2016).

[54] B. Deng, J. Wang, X. Wei, H. Yu, and H. Li, Phys. Rev. E 89, 062916 (2014).

[55] M. Borromeo and F. Marchesoni, Phys. Rev. E 73, 016142 (2006)

[56] B. S. Dandogbessi, O. Akin-Ojo, and A. Kenfack, Phys. Scrip. 90, 055206 (2015).

[57] S. Rajasekar, K. Abirami, and M. A. F. Sanjuan, Chaos 21, 033106 (2011).

[58] M. Imboden and P. Mohanty, Physics Reports 534, 89 (2014).

[59] A. Cleland, New J. Phys. 7, 787 (2005).

[60] U. Parlitz and W. Lauterborn, Phys. Lett. A 107A, 351 (1985).

[61] J. Kozłowski, U. Parlitz, and W. Lauterborn, Phys. Rev. E 51, 1861 (1995)

[62] C. Jeevarathinam, S. Rajasekar, and M. Sanjuan, Chaos 23, 013136 (2013) 